\title{
Mean first-passage times for systems driven by equilibrium persistent-periodic dichotomous noise
}

\author{
Josep M. Porrà and Katja Lindenberg \\ Department of Chemistry and Biochemistry, University of California, San Diego, La Jolla, California 92093-0340
}

(Received 10 November 1994)

\begin{abstract}
In a recent paper, [J. M. Porrà, J. Masoliver, and K. Lindenberg, Phys. Rev. E 48, 951 (1993)], we derived the equations for the mean first-passage time for systems driven by the coin-toss square wave, a particular type of dichotomous noisy signal, to reach either one of two boundaries. The coin-toss square wave, which we here call periodic-persistent dichotomous noise, is a random signal that can only change its value at specified time points, where it changes its value with probability $q$ or retains its previous value with probability $p=1-q$. These time points occur periodically at time intervals $\tau$. Here we consider the stationary version of this signal, that is, "equilibrium" periodicpersistent noise. We show that the mean first-passage time for systems driven by this stationary noise does not show either the discontinuities or the oscillations found in the case of nonstationary noise. We also discuss the existence of discontinuities in the mean first-passage time for random one-dimensional stochastic maps.
\end{abstract}

PACS number(s): 05.40. $+\mathrm{j}, 05.60 .+\mathrm{w}, 05.20 . \mathrm{Dd}, 05.90 .+\mathrm{m}$

\section{INTRODUCTION}

Noises that consist of signals that are randomized in some way at fixed time intervals are ubiquitous in theory and in practice. One particularly simple example is periodic dichotomous noise. This noise can assume only two values $a$ and $b$. One or the other value is chosen at periodic time intervals and the signal remains constant in between. The transitions between values occur with period $\tau$ and are characterized by a transition matrix

$$
\left(\begin{array}{ll}
p_{a a} & p_{a b} \\
p_{b a} & p_{b b}
\end{array}\right)
$$

that contains the probabilities $p_{i j}$ of a transition from value $i$ to value $j, i, j=\{a, b\}[1]$.

Different transition matrices have been considered in the literature. For instance, the transition matrix

$$
\left(\begin{array}{ll}
p & 1-p \\
p & 1-p
\end{array}\right)
$$

was treated a few years ago in studies of the stationary probability distribution of a linear system driven by dichotomous noise [2]; such a system had also been studied in a variety of other contexts [3]. The matrix

$$
\left(\begin{array}{cc}
p & 1-p \\
1-p & p
\end{array}\right)
$$

was used in the context of $R C$ low-pass filters [4]. This driving noise was called a "coin-toss square wave" and has the interesting property that it introduces a correlation between successive values of the noise. Recently, it has been applied to study the influence of noise in the logistic map [5].

These noises have also been considered indirectly in many contexts dealing with stochastic maps: The evo- lution of a system driven by a coin-toss square wave is described by two dynamics, each one corresponding to one of the values of the noise. Because the noise does not change during a period $\tau$, the state $X_{t}$ of the system at a time $t$ when the noise just had the opportunity to change and the state $X_{t+\tau}$ at time $t+\tau$ define a map for each value of the noise

$$
X_{t+\tau}=h_{\sigma}\left(X_{t}\right)
$$

where $\sigma=\{a, b\}$. Thus the evolution of the system at times that are multiples of $\tau$ is given by a stochastic map. These maps have found many applications in statistical mechanics, such as in one-dimensional random field Ising models and neural networks [6].

In a recent paper [7], we studied the mean first-passage time (MFPT), that is, the time that it takes a random variable to reach either of two assigned values for the first time, for systems driven by the coin-toss square wave. In this paper we call this noise "periodic-persistent dichotomous noise" because it belongs to the general class of noises defined above. The added name "persistent" comes from the fact that the transition matrix (3) is identical to the one found in the context of persistent random walks [8]. The system variable $x(t)$ satisfies the stochastic equation

$$
\dot{x}(t)=F(x(t), \eta(t)) .
$$

A deterministic dynamic is thus associated with each of the two values $a$ and $b=-a$ of the noise $\eta(t)$. We found the equations satisfied by the MFPT and were able to obtain the exact analytic solution for the driftless case

$$
F(x(t), \eta(t))=\eta(t) .
$$

For the linear case

$$
F(x(t), \eta(t))=\mu x(t)+\eta(t),
$$


we developed approximate solutions and checked them against simulations.

In [7], we showed that in both of these cases the MFPT as a function of the initial position $x(t=0)$ exhibits discontinuities that can be alternately positive and negative. This alternation of signs produces a remarkable nonsmooth oscillatory behavior in the MFPT as a function of the initial position. The reason for such striking behavior lies in the underlying periodicity of the noise or, in other words, in the fact that the noise is not stationary. The first opportunity for the noise to change its value comes exactly at time $\tau$. If the process starts sufficiently close to a barrier and moves toward it, then it will cross that barrier with certainty before time $\tau$; if, on the other hand, the starting point is not sufficiently close to the barrier for this to occur, then at time $\tau$ the noise may change the direction of the process and the barrier crossing may not occur during the next time interval $\tau$. This causes the MFPT to be discontinuous.

It is interesting to consider the situation when the periodic-persistent dichotomous noise is stationary (we call this noise "equilibrium" periodic persistent), that is, when the time to the first occasion that the noise may switch is a random variable [1]. The equations satisfied by the MFPT for the system driven by equilibrium noise differ from those we found in our previous work. In particular, herein we derive these equations and show that their solutions are continuous functions of the initial position.

In Sec. II we establish the equations satisfied by the first-passage-time probability density to reach either of two boundaries for a general periodic-persistent dichotomous noise when the time to the first occasion that the noise may switch its value is random. In Sec. III the discontinuities shown by the solution of the equations for the coin-toss square signal are discussed. In Sec. IV we concentrate on the equilibrium periodic-persistent noise and show that the discontinuities in the mean first-passage time disappear. Brief conclusions are drawn in Sec. V.

\section{FIRST PASSAGE-TIME EQUATIONS}

At any one time our system is driven by one of two different dynamics, each one associated with one value of the noise. Let $F_{+}(x) \equiv F(x, \eta(t)=a)$ and $F_{-}(x) \equiv$ $F(x, \eta(t)=-a)$ denote these dynamics when $\eta(t)=+a$ and $\eta(t)=-a$, respectively. We are interested in systems that are able to reach either of two boundaries. Consequently, if $x_{0}$ is the initial position of the process, we suppose that $F_{+}\left(x_{0}\right)$ and $F_{-}\left(x_{0}\right)$ have different signs, e.g., we specifically consider the case where $F_{+}\left(x_{0}\right)>0$ and $F_{-}\left(x_{0}\right)<0$. We further consider only systems that evolve toward asymptotically fixed stable points under the $F_{ \pm}$dynamics, specifically toward $x_{s}^{+}$ when evolving under $F_{+}(x)$ and $x_{s}^{-}$when under $F_{-}(x)$. Hence $F_{+}\left(x_{s}^{+}\right)=0$ and $F_{-}\left(x_{s}^{-}\right)=0$. Moreover, we assume that

$$
\begin{array}{ll}
F_{+}(x)>0 & \text { if } x_{s}^{-} \leq x<x_{s}^{+} \\
F_{-}(x)<0 & \text { if } x_{s}^{-}<x \leq x_{s}^{+} .
\end{array}
$$

In this way, if the system is initially between the two "natural barriers" $x_{s}^{+}$and $x_{s}^{-}$, then the system remains there forever. Therefore, if we calculate the first-passage time for the process to reach certain values, say, $z_{1}$ or $z_{2}$, these values must lie between the two natural barriers, i.e.,

$$
x_{s}^{-}<z_{1}<z_{2}<x_{s}^{+} .
$$

Let us now define some quantities that will be useful later. We call $\tau^{+}$the time for the system leaving from $x_{0}$ to reach the boundary $z_{2}$ when driven by $F_{+}(x) . \tau^{-}$ is defined analogously for $z_{1}$ and $F_{-}(x)$. From Eq. (5), it follows that

$$
\tau^{+}=\int_{x_{0}}^{z_{2}} \frac{d x}{F_{+}(x)}, \quad \tau^{-}=\int_{x_{0}}^{z_{1}} \frac{d x}{F_{-}(x)} .
$$

The new position $x(t)$ after the system has evolved for a time $t$ under the dynamic $F_{+}(x)\left[F_{-}(x)\right]$ from $x_{0}$ is $x^{+}\left(t, x_{0}\right)\left[x^{-}\left(t, x_{0}\right)\right]$, that is,

$$
t=\int_{x_{0}}^{x^{+}\left(t, x_{0}\right)} \frac{d x}{F_{+}(x)}, \quad t=\int_{x_{0}}^{x^{-}\left(t, x_{0}\right)} \frac{d x}{F_{-}(x)} .
$$

Successive positions after each period of time $\tau$, $x^{ \pm}\left(t, x_{0}\right)$, define two maps

$$
x_{n+1}=x^{ \pm}\left(\tau, x_{n}\right) \equiv h^{ \pm}\left(x_{n}\right)
$$

in the interval $\left[x_{s}^{-}, x_{s}^{+}\right]$, as discussed in the Introduction, Eq. (4). From the properties of the dynamics $F_{ \pm}(x)$, Eq. (8), it follows that both maps are monotonic in that interval. $x_{s}^{+}$is a fixed point of the map $h^{+}$and $x_{s}^{-}$of $h^{-}$. The integration of Eq. (5) over a period $\tau$ gives a one-dimensional stochastic map that consists of random switches between the maps $h^{+}$and $h^{-}$. The matrix (1) gives the probability for different transitions. The inverse of the map $h^{+}\left[h^{-}\right]$, where it exists, will be denoted by $g^{+}\left[g^{-}\right]$. The functions $g^{ \pm}$are defined implicitly as

$$
\tau=\int_{g^{+}(x)}^{x} \frac{d x^{\prime}}{F_{+}\left(x^{\prime}\right)}, \quad \tau=\int_{g^{-}(x)}^{x} \frac{d x^{\prime}}{F_{-}\left(x^{\prime}\right)}
$$

and give the position from where it takes the system a time $\tau$ to reach $x$ evolving under dynamics $F_{ \pm}(x)$ or, in other words, the preimage of $x$ under $h^{ \pm}$. The different dynamical quantities defined above are sketched in Fig. 1

We next define $p_{+}^{m}\left(t, x_{0}\right)$ and $p_{-}^{m}\left(t, x_{0}\right)$ to be, respectively, the first-passage time probability density to reach either of the values $z_{1}$ and $z_{2}$ if initially $\eta(0)=+a$ and $\eta(0)=-a$. The superscript $m$ is an explicit reminder that the time to the first opportunity for the noise to switch may be a random variable, with probability density function $\psi_{1}(t)$. The periodic-persistent dichotomous noise, or coin-toss square signal, is recovered with the choice $\psi_{1}(t)=\delta(t-\tau)$. In this paper, we are interested in the choice of $\psi_{1}(t)$ that causes the noise to be stationary. Results for this specific choice will be indicated by the subscript eq. In the Appendix, it is shown that the 


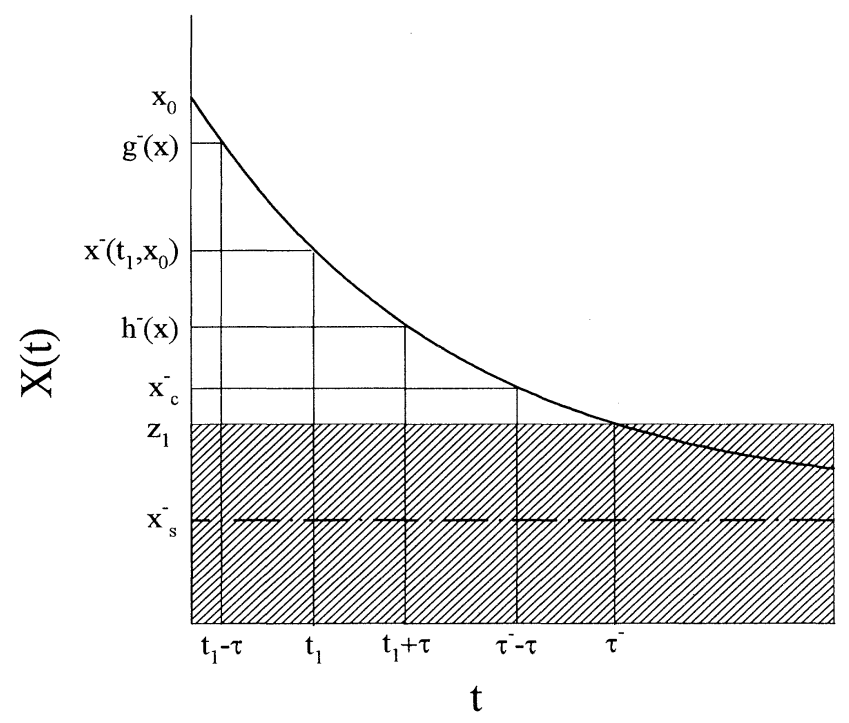

FIG. 1. Dynamical quantities defined in Sec. III when the driving noise $\eta(t)=-a$. The trajectory $x^{-}\left(t, x_{0}\right)$ approaches its fixed point $x_{s}^{-}$. The position at time $t_{1}$ is denoted by $x^{-}\left(t_{1}, x_{0}\right)$. The functions $h^{-}(x)$ and $g^{-}(x)$ are defined in the text. The shaded region corresponds to the region outside the boundaries $z_{1}$ and $z_{2}$.

appropriate choice is the uniform distribution between 0 and $\tau$.

The equation satisfied by $p_{+}^{m}\left(t, x_{0}\right)$ is

$$
\begin{aligned}
p_{+}^{m}\left(t, x_{0}\right)= & \delta\left(t-\tau^{+}\right) \Psi_{1}(t) \\
& +\int_{0}^{\tau^{+}}\left[p p_{+}^{o}\left(t-t^{\prime}, x^{+}\left(t^{\prime}, x_{0}\right)\right)\right. \\
& \left.+q p_{-}^{o}\left(t-t^{\prime}, x^{+}\left(t^{\prime}, x_{0}\right)\right)\right] \psi_{1}\left(t^{\prime}\right) d t^{\prime},
\end{aligned}
$$

where $\Psi_{1}(t)$ is the survival probability associated with $\psi_{1}(t)$

$$
\Psi_{1}(t)=\int_{t}^{\infty} \psi_{1}\left(t^{\prime}\right) d t^{\prime}
$$

The superscript $o$ indicates that the next opportunity for the noise to change comes exactly at a time $\tau$ after the previous change. The first term on the right-hand side accounts for the probability that $z_{2}$ is reached at time $t$ before the noise has an opportunity to change from its initial value. If the noise does change before the process first reaches either boundary at time $t$, it does so at some time $t^{\prime}<t$. After this change, the noise becomes a periodic-persistent dichotomous process that can reach a boundary in the next time interval $\tau^{+}$. This possibility is embodied in the remainder of the right-hand side of the equation. The equation for $p_{-}^{m}\left(t, x_{0}\right)$ is obtained by interchanging the indices (superscripts and subscripts) + and - in Eq. (14). In order to calculate the probability densities $p_{ \pm}^{o}$ in Eq. (14), we simply need to choose

$$
\psi_{1}(t)=\delta(t-\tau)
$$

that is, the noise then is periodic-persistent dichotomous.
The result, with the superscript $m$ replaced by $o$, is a closed set of equations for the $p^{o}$ 's. Once this system is solved, the solution (14) can be obtained. The equations for the first-passage time probability density of a system driven by a periodic noise with transition matrix given by Eq. (1) can be written in a similar way.

An integral equation for the MFPT follows from the relation

$$
T^{ \pm}\left(x_{0}\right)=-\left.\frac{\partial}{\partial s} \hat{p}_{ \pm}\left(s, x_{0}\right)\right|_{s=0},
$$

where the superscripts + and - on $T$ respectively distinguish the cases where $\eta(0)=+a$ and $\eta(0)=-a$. Here $\hat{p}_{ \pm}\left(s, x_{0}\right)$ represents the Laplace transform of $p_{ \pm}\left(t, x_{0}\right)$. A subscript will be attached to $T$ to differentiate between the MFPT's for various $\psi_{1}(t)$.

From Eq. (17), we find

$$
\begin{aligned}
T_{m}^{+}\left(x_{0}\right)= & \int_{0}^{\tau^{+}} \Psi_{1}\left(t^{\prime}\right) d t^{\prime} \\
& +\int_{0}^{\tau^{+}}\left[p T_{o}^{+}\left(x^{+}\left(t^{\prime}, x_{0}\right)\right)+q T_{o}^{-}\left(x^{+}\left(t^{\prime}, x_{0}\right)\right)\right] \\
& \times \psi_{1}\left(t^{\prime}\right) d t^{\prime}
\end{aligned}
$$

The interchange of superscripts + and - gives an equivalent equation for $T_{m}^{-}\left(x_{0}\right)$. Thus $T_{\mathbf{e q}}^{ \pm}\left(x_{0}\right)$ and, in general, $T_{m}^{ \pm}\left(x_{0}\right)$ can be obtained from the results for the MFPT for the system driven by periodic-persistent dichotomous noise. The relation between the MFPT of a system driven by dichotomous noise of arbitrary statistics and the MFPT when it is driven by the stationary version of the same dichotomous noise was proved in general in a recent paper [9].

The equation for $T_{o}^{+}\left(x_{0}\right)$ derived from Eq. (18), with $\psi_{1}(t)=\delta(t-\tau)$, is equivalent to the one we found previously [7]. However, the notation used earlier was slightly different because there we wrote a separate equation for the boundary condition while here it is included in Eq. (18). In fact, with $\psi_{1}(t)=\delta(t-\tau)$, Eq. (18) reads

$$
T_{o}^{+}\left(x_{0}\right)=\tau+p T_{o}^{+}\left(x^{+}\left(\tau, x_{0}\right)\right)+q T_{o}^{-}\left(x^{+}\left(\tau, x_{0}\right)\right)
$$

if $z_{1} \leq x_{0} \leq x_{c}^{+}$and

$$
T_{o}^{+}\left(x_{0}\right)=\int_{x_{0}}^{z_{2}} \frac{d x}{F_{+}(x)}
$$

if $x_{c}^{+}<x_{0} \leq z_{2}$. The value of $x_{0}$ that makes $\tau^{+}$greater than $\tau$ is the solution of $z_{2}=x^{+}\left(\tau, x_{0}\right)$ and is denoted by $x_{c}^{+}$. Equivalently, $x_{c}^{+}$is the preimage of $z_{2}$ under $h^{+}$. Equation (20) was considered in our previous paper as a boundary condition. Analogous reasoning leads to the equations for $T_{o}^{-}\left(x_{0}\right)$,

$$
T_{o}^{-}\left(x_{0}\right)=\tau+p T_{o}^{-}\left(x^{-}\left(\tau, x_{0}\right)\right)+q T_{o}^{+}\left(x^{-}\left(\tau, x_{0}\right)\right)
$$

if $x_{c}^{-} \leq x_{0} \leq z_{2}$ and

$$
T_{o}^{-}\left(x_{0}\right)=\int_{x_{0}}^{z_{1}} \frac{d x}{F_{-}(x)}
$$


if $z_{1} \leq x_{0}<x_{c}^{-}$, where $x_{c}^{-}$is given implicitly by $z_{1}=x^{-}\left(\tau, x_{c}^{-}\right)$. Before considering the mean firstpassage time $T_{\text {eq }}^{ \pm}\left(x_{0}\right)$ for the stationary process, we first discuss in the next section why $T_{o}^{ \pm}\left(x_{0}\right)$ is, in general, a discontinuous function.

\section{DISCONTINUITIES}

In our previous paper [7], we discussed the appearance of discontinuities in the MFPT as a function of initial position in the driftless case $\dot{x}(t)=\eta(t)$ and also in the linear case $\dot{x}(t)=\mu x(t)+\eta(t)$. The discontinuities occur because there is an initialization region for the random process $x(t)$ around the boundaries $z_{1}$ and $z_{2}$ where the first-passage time to the boundaries is not random. We established the occurrence of the discontinuities analytically in the driftless case and via simulations in the linear case and we also showed in the latter case that the value of the largest discontinuity is in agreement with a calculation based on Eqs. (19)-(22). However, we considered neither the total number of discontinuities nor the values of those besides the largest. Here we show that the set of discontinuities is infinite in general and we also establish how discontinuities at various different initial values are related to one another.

Let us begin with discontinuities in $T_{o}^{-}\left(x_{0}\right)$. In the region $x_{0} \in\left[z_{1}, x_{c}^{-}\right), T_{o}^{-}\left(x_{0}\right)$ is deterministic and its value is given by Eq. ( 22). However, when $x_{0}>x_{c}^{-}, T_{o}^{-}\left(x_{0}\right)$ becomes a random variable because, after a time $\tau$, the noise may switch and make the system go away from $z_{1}$. Moreover, the change of the first-passage time to a boundary from deterministic to random behavior in an infinitesimal region around $x_{c}^{-}$causes $T_{o}^{-}\left(x_{c}^{-}\right)$to be discontinuous. When $x_{0}$ is sufficiently close to but smaller than $x_{c}^{-}, T_{o}^{-}\left(x_{0}\right)$ is essentially equal to $\tau$. However, if $x_{0}$ is close to but larger than $x_{c}^{-}$, the system exits the interval at time $\tau$ only with probability $p$, while with probability $q$, it goes away from $z_{1}$ and remains in the interval. If this occurs, it takes at least an additional time $\tau$ for the noise to switch again and for the process to go back to the barrier $z_{1}$ (unless it has crossed the other barrier in the meantime). Thus the discontinuity at $x_{c}^{-}$is greater than $q \tau$.

More precisely, from Eqs. (21) and (22), we find the value of the discontinuity at $x_{c}^{-}$to be

$$
\Delta T_{o}^{-}\left(x_{c}^{-}\right)=q T_{o}^{+}\left(z_{1}\right),
$$

where we have defined

$$
\Delta T_{o}^{-}(y)=\lim _{\epsilon \rightarrow 0^{+}}\left[T_{o}^{-}(y+\epsilon)-T_{o}^{-}(y-\epsilon)\right] .
$$

It should be pointed out that in general $T_{o}^{-}\left(x_{0}\right)$ is continuous from the right at point $x_{c}^{-}$, that is,

$$
T_{o}^{-}\left(x_{c}^{-}\right)=\lim _{\epsilon \rightarrow 0^{+}} T_{o}^{-}\left(x_{c}^{-}+\epsilon\right) \text {. }
$$

Next we consider a point $y$ at which $T_{o}^{-}$and/or $T_{o}^{+}$are discontinuous. Then, if $z_{1}<y^{\prime}<z_{2}$ with $y^{\prime}=g^{-}(y), T_{o}^{-}$ is also discontinuous at $y^{\prime}$. We recall that the $g^{ \pm}$were defined earlier and give the position from where it takes the system a time $\tau$ to reach point $y$. The value of the discontinuity follows from Eq. (21),

$$
\Delta T_{o}^{-}\left(y^{\prime}\right)=p \Delta T_{o}^{-}(y)+q \Delta T_{o}^{+}(y) .
$$

Similarly, if $y^{\prime \prime}=g^{+}(y)$ is within the interval $\left(z_{1}, z_{2}\right), T_{o}^{+}$ has a discontinuity at $y^{\prime \prime}$,

$$
\Delta T_{o}^{+}\left(y^{\prime \prime}\right)=p \Delta T_{o}^{+}(y)+q \Delta T_{o}^{-}(y) .
$$

Earlier we demonstrated that $T_{o}^{-}$is discontinuous at $x_{c}^{-}$. Therefore, successive images of that point under $g^{ \pm}$, as long as they are in $\left(z_{1}, z_{2}\right)$, become points of discontinuity. In other words, preimages of $x_{c}^{-}$that are in $\left(z_{1}, z_{2}\right)$ develop into discontinuity points. If a point $x_{0}$ in $\left(z_{1}, z_{2}\right)$ can be expressed as the result of successive applications of $g^{ \pm}$

$$
x_{0}=g^{+} \circ g^{\alpha_{n-1}} \circ \cdots \circ g^{\alpha_{1}}\left(x_{c}^{-}\right) \text {, }
$$

where $\alpha_{i}=\{+,-\}, n=\{1,2, \ldots\}$, and all intermediate points

$$
y=g^{\alpha_{j}} \circ \cdots \circ g^{\alpha_{1}}\left(x_{c}^{-}\right)
$$

with $j=\{1, \ldots, n-1\}$ are in $\left(z_{1}, z_{2}\right)$, then $T_{o}^{+}(x)$ is discontinuous from the left at $x=x_{0}$. Similarly, if

$$
x_{0}=g^{-} \circ g^{\alpha_{n-1}} \circ \cdots \circ g^{\alpha_{1}}\left(x_{c}^{-}\right)
$$

with the same conditions as above, then $T_{o}^{-}(x)$ is discontinuous from the left at $x=x_{0}$.

Following identical reasoning as that which led to Eq. (23) leads to the conclusion that $T^{+}\left(x_{0}\right)$ is discontinuous from the right at $x_{c}^{+}$and that

$$
\Delta T_{o}^{+}\left(x_{c}^{+}\right)=q T_{o}^{-}\left(z_{2}\right)
$$

where $\Delta T_{o}^{+}$is defined as in Eq. (24). This discontinuity will also generate others at points

$$
x_{0}=g^{\alpha_{n}} \circ g^{\alpha_{n-1}} \circ \cdots \circ g^{\alpha_{1}}\left(x_{c}^{+}\right),
$$

$n=\{1,2, \ldots\}$, as long as points $y=g^{\alpha_{j}} \circ \cdots \circ g^{\alpha_{1}}\left(x_{c}^{+}\right)$ belong to $\left(z_{1}, z_{2}\right)$. If $\alpha_{n}=+$, the discontinuity is in $T_{o}^{+}$; otherwise it is in $T_{o}^{-}$.

We have thus demonstrated that, in general, $T^{ \pm}$has an infinite number of discontinuities in the interval $\left(z_{1}, z_{2}\right)$ and that they are related through Eqs. (26) and (27). A finite number of discontinuities arises when, after a certain number of iterations of $g^{+}$and $g^{-}$, there are no more preimages inside the interval $\left(z_{1}, z_{2}\right)$. A necessary condition for this to happen is that there exists a set inside the interval whose preimage, under both $g^{+}$and $g^{-}$, is outside $\left(z_{1}, z_{2}\right)$. We say in this case that $g^{+}$and $g^{-}$are nonoverlapping in $\left(z_{1}, z_{2}\right)$. In fact, if $h^{ \pm}$are nonoverlapping in $\left(x_{s}^{-}, x_{s}^{+}\right)$, i.e., there are points in $\left(x_{s}^{-}, x_{s}^{+}\right)$that are not images of any point in that interval, then $g^{ \pm}$are nonoverlapping for any $z_{1}, z_{2}$. On the other hand, if $h^{+}$ and $h^{-}$do overlap, $g^{ \pm}$become nonoverlapping or overlapping as $z_{1}$ and $z_{2}$ vary. This leads to the existence of critical values of $z$ where there is a transition from a fi- 
nite to an infinite number of discontinuities in the MFPT as a function of $x_{0}$.

Preimages of $x_{c}^{-}$generate discontinuities from the left while those produced from $x_{c}^{+}$are discontinuities from the right. A singular case arises when a point is a preimage of both $x_{c}^{-}$and $x_{c}^{+}$. Then, successive images of this point give points where $T_{o}^{+}$or $T_{o}^{-}$are discontinuous from both sides.

The driftless case provides an example of such a singular case. Let us consider the MFPT to $(0, L)$ for the driftless system (6). We take $\eta(t)= \pm 1$ and $\tau=1$. With this, $F_{ \pm}= \pm 1$ and $h^{ \pm}(x)=x \pm 1$. The inverse map is $g^{ \pm}=x \mp 1$. Thus $x_{c}^{-}=1$ and $x_{c}^{+}=L-1$. Successive images of $x_{c}^{-}$under $g^{ \pm}$lead to discontinuities at the set $\Omega_{1}=\{1,2, \ldots, N\}$, where $N$ is the integer part of $L$. On the other hand, $x_{c}^{+}$generates discontinuities at points $\Omega_{2}=\{L-1, L-2, \ldots, L-N\}$. If $L$ is not an integer, both $T_{o}^{+}$and $T_{o}^{-}$are continuous from the right at points $\Omega_{1}$, while at points $\Omega_{2}$ they are continuous from the left. However, when $L$ is an integer, $\Omega_{1}$ and $\Omega_{2}$ coincide and any integer between 1 and $L-1$ is an image of both $x_{c}^{-}$ and $x_{c}^{+}$. Therefore, at these points discontinuities from the right and from the left collapse and lead to isolated discontinuous values for the MFPT [7]. This effect is seen in Fig. 2, where we plot $T_{o}\left(x_{0}\right)$, the MFPT averaged over the initial value of the driving noise,

$$
T_{o}\left(x_{0}\right)=\frac{T_{o}^{+}\left(x_{0}\right)+T_{o}^{-}\left(x_{0}\right)}{2} .
$$

The isolated dots are the values of $T_{o}\left(x_{0}\right)$ at integer values of $x_{0}$. Note that at these points the quantity $\Delta T_{o}\left(x_{0}\right)$ defined in Eq. (24) does not vanish except at $x_{0}=0$.

Another property that follows from Eqs. (26) and (27) is that successive preimages of the point $x_{c}^{-}$(or $x_{c}^{+}$) generate discontinuities that are consecutively diminished by a factor $p$ or $q$. Thus the greater (the minimum) $n$ is in the expression of a point $x_{0}$ as an image of $x_{c}^{-}$or $x_{c}^{+}$, Eqs. (28)-(32), the smaller the value of the discontinuity at that point. When $\tau$ goes to zero, the discontinuity at

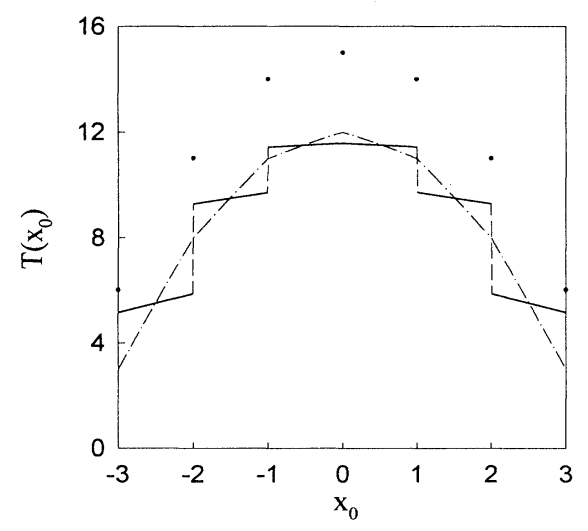

FIG. 2. Mean first-passage time to $z_{1}=-3$ and $z_{2}=3$ for the driftless case, with $a=1, \tau=1$, and $p=0.5$, driven by the two noises considered: (i) the periodic-persistent dichotomous noise $T_{o}\left(x_{0}\right)$ (solid line together with the circle symbols) and (ii) the equilibrium periodic-persistent noise $T_{\text {eq }}\left(x_{0}\right)$ (dot-dashed line). $x_{c}^{-}$(and similarly at $x_{c}^{+}$) goes to zero provided that

$$
q_{\tau \rightarrow 0}=0
$$

or

$$
T^{+}\left(z_{1}\right) \rightarrow 0
$$

In both cases, the MFPT becomes a smoother function of $x_{0}$. Incidentally, we remark that conditions (34) and (35) correspond to the two limits of the periodicpersistent dichotomous noise (see the Appendix). When $q \rightarrow 0$, periodic-persistent dichotomous noise converges (in distribution) to a Markovian dichotomous noise, while the case $T^{+}\left(z_{1}\right) \rightarrow 0$ corresponds to the limit in which periodic-persistent dichotomous noise becomes equivalent to white noise.

For the linear case, Eq. (7), we have not been able to obtain analytic solutions of Eqs. (19)-(22). However, values obtained by direct simulation do satisfy Eqs. (26) and (27) within the random errors inherent in Monte Carlo methods. We consider the MFPT to $z_{1}=-1$ and $z_{2}=1$ for a linear system with $\mu=0.5$. The noise again takes the values $\pm 1, \tau$ is set to 0.1 , and $p=q=0.5$. Therefore, $F_{ \pm}(x)=0.5 x \pm 1$ and

$$
h^{ \pm}(x)=x b \pm 2(1-b),
$$

where

$$
b=\exp (-\mu \tau)=0.95122 .
$$

Preimages are given by

$$
g^{ \pm}(x)=x / b \mp 2(1 / b-1) .
$$

Thus $x_{c}^{-}=g^{-}\left(z_{1}\right)=-0.9487$ and $x_{c}^{+}=g^{+}\left(z_{2}\right)=$ 0.9487. In Fig. 3, the results of the simulation for $T_{o}\left(x_{0}\right)$, the mean first-passage time averaged over initial values of the noise are shown for $x_{0}$ between -1 and 0 . The error bars of the simulation are of the order of the size of the symbols used in the figure. First, Eq. (23) is checked, taking into account that $T_{o}^{+}\left(z_{1}\right)=0$ and that the discontinuity of $T_{o}^{+}\left(x_{c}^{-}\right)$, if it exists, will be much smaller than that of $T_{o}^{-}\left(x_{c}^{-}\right)$. As explained earlier, $T_{o}^{+}\left(x_{0}\right)$ is discontinuous at $x_{c}^{-}$if this point results from successive applications of $g^{ \pm}$on either $x_{c}^{+}$, Eq. (32), or $x_{c}^{-}$, Eq. (28). In the first case, $x_{c}^{-}$is a preimage of $x_{c}^{+}$,

$$
x_{c}^{-}=g^{+} \circ g^{\alpha_{n-1}} \circ \cdots \circ g^{+}\left(x_{c}^{+}\right) \text {, }
$$

and, from Eq. (38), it can be shown that the minimum number $n$ of times that $g^{ \pm}$is applied to $x_{c}^{+}$to give $x_{c}^{-}$ is greater than $n_{1}=22$. Therefore, if $x_{c}^{+}$generates a discontinuity at $x_{c}^{-}$, it will be $p^{-22}$ times smaller than $\Delta T_{o}\left(x_{c}^{-}\right)$. On the other hand, if $x_{c}^{-}$is obtained as a preimage of itself by alternative application of $g^{+}$and $g^{-}$, it will generate a discontinuity in $T_{o}^{+}\left(x_{0}\right)$ at $x_{c}^{-}$. However, $n$ in this case will be greater than the number of times $n_{2}$ that $g^{-}$has to be applied to $x_{c}^{-}$in order to give a value greater than $h^{+}\left(x_{c}^{-}\right)$. From Eq. (38), it follows that $n_{2}=3$. In fact, we numerically generated all preimages of $x_{c}^{-}$and $x_{c}^{+}$up to $n=20$ and checked that 


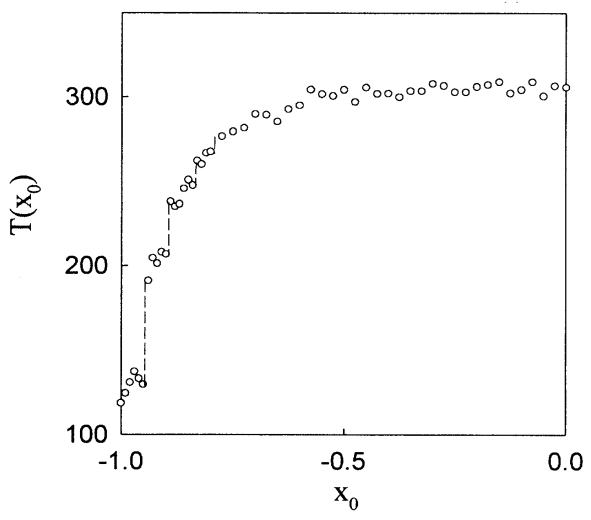

FIG. 3. Simulation results for the mean first-passage time $T_{o}\left(x_{0}\right)$ to $z_{2}=-z_{1}=1$ as a function of $x_{0}$ for the linear system (7) with $\mu=0.5$. The parameters of the noise are $a=1$, $\tau=1$, and $p=0.5$. The dashed lines show the discontinuities of $T_{o}\left(x_{0}\right)$ at $x_{0}=x_{c}^{-}=-0.949, x_{0}=-0.895,-0.838,-0.779$. The value of the discontinuities at these points is related to the value of $T_{o}\left(z_{1}\right)$.

$x_{c}^{-}$does not become a preimage of either $x_{c}^{-}$or $x_{c}^{+}$for $n<20$. We thus take

$$
\Delta T_{o}\left(x_{c}^{-}\right) \simeq \frac{\Delta T_{o}^{-}\left(x_{c}^{-}\right)}{2}=\frac{q T_{o}^{+}\left(z_{1}\right)}{2}=q T_{o}\left(z_{1}\right)
$$

Upon substitution of the numerical values $\Delta T_{o}\left(x_{c}^{-}\right)=61$ and $T_{o}\left(z_{1}\right)=119$, it is proved that Eq. (23) is satisfied within the errors of the simulation. The discontinuities at the next three preimages of $x_{c}^{-}, x_{0}=$ $-0.8948,-0.8382,-0.7786$, shown in Fig. 3 by dashed lines, are related through Eq. (26). The value of the discontinuities of $T_{o}^{+}$at these points is not considered for the same reason that $\Delta T_{o}^{+}\left(x_{c}^{-}\right)$was disregarded. Effectively, it follows from our simulation results that

$$
\begin{aligned}
\Delta T_{o}(-0.7786) & =\frac{1}{2} \Delta T_{o}(-0.8382) \\
& =\frac{1}{4} \Delta T_{o}(-0.8948)=\frac{1}{8} \Delta T_{o}\left(x_{c}^{-}\right) .
\end{aligned}
$$

The maps $h^{ \pm}$, Eq. (36), overlap in the sense defined earlier if the parameter $b>1 / 2$ because then $h^{+}\left(x_{s}^{-}\right)<0$. The value of $b$ considered in the example, Eq. (37), satisfies this condition. Therefore, a critical $z, z_{c}$, exists where the number of discontinuities changes from finite to infinite. Taking $z_{1}=-z_{2}=-z$, the critical $z$ reads

$$
z_{c}=2 \frac{1-b}{b}=0.102542 \text {. }
$$

If $z<z_{c}$, the MFPT time as a function of the initial position shows a finite number of discontinuities; if $z>$ $z_{c}$, this number becomes infinite.

\section{STATIONARY NOISE}

In Sec. II we showed that once $T_{o}^{ \pm}\left(x_{0}\right)$ is known, the MFPT $T_{m}^{ \pm}\left(x_{0}\right)$ for a system driven by a general periodic- persistent noise dichotomous can be obtained by integration [cf. Eq. (18)]. Because of the integration, the behavior of $T_{m}^{ \pm}\left(x_{0}\right)$ is smoother than that of $T_{o}^{ \pm}\left(x_{0}\right)$ [provided that $\psi_{1}(t)$ does not contain $\delta$ functions] and the discontinuities fade. This holds, in particular, for $T_{\mathrm{eq}}^{ \pm}\left(x_{0}\right)$. The oscillatory behavior also disappears because it was a consequence of the alternation of discontinuities of different signs.

We recall here the expression for $T_{o}^{+}\left(x_{0}\right)$ to escape the interval $(0, L)$ for the driftless case: in dimensionless units $(a=1, \tau=1)[7]$,

$$
T_{o}^{+}\left(x_{0}\right)=L-x_{0}+A\left(x_{0}\right) j-\xi j^{2},
$$

where

$$
A\left(x_{0}\right)=\left\{\begin{array}{c}
N \xi+\frac{\xi}{1+N \xi}\left[2\left(x_{0}+j\right)-L-N+1\right] \\
\quad \text { if } L-(j+1)<x_{0}<N-j \\
(N+1) \xi+\frac{\xi}{1+(N+1) \xi}\left[2\left(x_{0}+j\right)-L-N\right] \\
\quad \text { if } N-j \leq x_{0} \leq L-j .
\end{array}\right.
$$

Here $j \equiv\left[L-x_{0}\right]$ is the integer part of $L-x_{0}$. The parameter $\xi$ is defined as

$$
\xi=\frac{q}{p} .
$$

$T_{o}^{-}\left(x_{0}\right)$ follows from the relation that applies in the driftless case,

$$
T_{o}^{-}\left(x_{0}\right)=T_{o}^{+}\left(L-x_{0}\right) .
$$

$T_{\text {eq }}^{+}\left(x_{0}\right)$ is obtained by using Eq. (40) in Eq. (18). Although it is a continuous function of the initial position, the analytical expression for $T_{\text {eq }}^{+}\left(x_{0}\right)$ is rather complicated because its first derivative is discontinuous at the same positions where $T_{o}^{ \pm}\left(x_{0}\right)$ is discontinuous. Thus it is not possible to avoid the use of the integer part and the final expression becomes complex. In Fig. 4, we plot $T_{\text {eq }}^{+}\left(x_{0}\right)$ obtained by numerical integration of Eq. (18) for some values of the parameters. The expression for $T_{o}^{+}$ simplifies when $L=N$, an integer, because discontinuities then occur only at integer values of $x_{0}$, as discussed in Sec. III. In this case, and for $p=q=0.5$,

$$
\begin{aligned}
T_{o}^{+}\left(x_{0}\right)= & \frac{2 N^{2}}{N+1}-\frac{N-1}{N+1} i^{2}+\frac{N^{2}-3 N+1}{N+1} i \\
& +\frac{N-3-2 i}{N+1} x_{0},
\end{aligned}
$$

if $x_{0}$ is not an integer. The integer part of $x_{0}$ is denoted by $i$. For integer values of $x_{0}, j=\{0, \ldots, N\}, T_{o}^{+}(j)$ coincides with the expression of the mean first-passage time for a persistent random walk on a lattice with absorbing boundaries at 0 and $N$ when the first step of the random walker is to the right [10],

$$
T_{o}^{+}(j)=2 N+(N-2) j-j^{2} .
$$

Substitution of Eq. (44) into Eq. (18) yields 


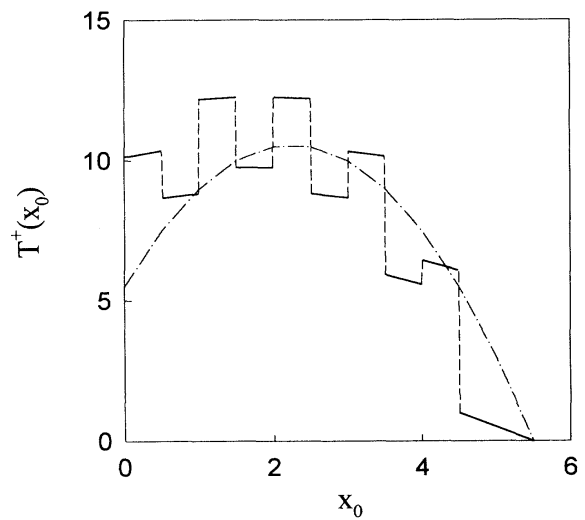

FIG. 4. Mean first-passage times $T_{o}^{+}\left(x_{0}\right)$ (solid line) and $T_{\text {eq }}^{+}\left(x_{0}\right)$ (dot-dashed line) to $z_{1}=0$ and $z_{2}=5.5$ for the driftless case with $a=1, \tau=1$, and $p=0.5$.

$$
\begin{aligned}
T_{\mathrm{eq}}^{+}\left(x_{0}\right)= & -\frac{1}{N+1} x_{0}^{2}+\frac{N^{2}-(1+2 i) N-1}{N+1} x_{0} \\
& +N+\frac{N}{N+1} i(i+1) .
\end{aligned}
$$

Note that $T_{o}^{+}(j)$ does not contribute to the integral in Eq. (18). Finally, the MFPT to exit the region $(-N, N)$ for $N$ integer in the driftless case with $p=q=0.5$ averaged over initial values of the noise is

$$
\begin{aligned}
T_{\text {eq }}\left(x_{0}\right)= & N^{2}+N+\frac{1}{2 N+1}[2 N j(j+1) \\
& \left.-x_{0}^{2}-2 N\left|x_{0}\right|(1+2 j)\right],
\end{aligned}
$$

with $j=\left[\left|x_{0}\right|\right]$, the integer part of the absolute value $x_{0}$. In Fig. 2, this result is plotted and compared with $T_{o}\left(x_{0}\right)$ for the same parameter values.

\section{CONCLUSIONS}

We have revisited the problem of the mean firstpassage time to either of two barriers for systems driven by periodic-persistent dichotomous noise. This noise takes on one of two values that can change with probability $q$ (or remain the same with probability $1-q$ ) only at prescribed periodic time intervals $\tau$. When $q \rightarrow 0$ the driving signal is essentially constant; when $q \rightarrow 1$ it is essentially periodic. If $\tau \rightarrow 0$ with $q=\lambda \tau$, the noise converges to the familiar Markovian dichotomous noise, and if $\tau \rightarrow 0$ as the magnitude of the noise $a \rightarrow \infty$ in a particular way, the signal converges to white noise. In general, this simple noise is a model for a signal that combines periodic and random components.

When the initial time $t=0$ is precisely a time at which the noise has an opportunity to change (the next opportunity occurring at time $t=\tau$ ), the noise is not stationary. In this case, the mean first-passage time as a function of the initial position of the process exhibits discontinuities and/or oscillations. Here we extend our earlier analysis of these unusual features. If the first opportunity for the value of the noise to change occurs with equal probability at any time within the interval $0<t<\tau$ (the next opportunity occurring at a time $\tau$ after the first possible change), the noise becomes stationary and no discontinuities and/or oscillations are observed in the mean first-passage time.

\section{ACKNOWLEDGMENTS}

This work has been supported in part by the Dirección General de Investigación Científica y Técnica under Contract No. DGCYT PB93-0812, by the Societat Catalana de Física (Institut d'Estudis Catalans), and by the U.S. Department of Energy Grant No. DE-FG03-86ER13606. Support of the Gaspar de Portola Catalonian Studies Program at the University of California is also gratefully acknowledged. One of us (J.M.P.) thanks the Department of Chemistry and Biochemistry at the University of California, San Diego for its kind hospitality and the Ministerio de Educación y Ciencia of Spain for financial support. We thank Professor Jaume Masoliver for a careful reading of the manuscript.

\section{APPENDIX: NOISE PROPERTIES}

The stationary noise associated with a periodicpersistent dichotomous noise is obtained by taking a specific probability density function, $\psi_{1}(t)$, for the time to the first opportunity that the noise can change its value. With this choice, the renewal process that generates the noise becomes an equilibrium renewal process [1] and the correlation function $C\left(t, t^{\prime}\right) \equiv\left\langle\eta(t) \eta\left(t^{\prime}\right)\right\rangle$ depends only on the difference $\left|t-t^{\prime}\right|$. The function $\psi_{1}(t)$ is related to the probability density function $\psi(t)$ of the time between switching opportunities of the noise by

$$
\psi_{1}(t)=\frac{1}{\mu} \int_{t}^{\infty} \psi\left(t^{\prime}\right) d t^{\prime},
$$

where $\mu$ is the first moment of $\psi(t)$. For the periodicpersistent dichotomous noise $\psi(t)=\delta(t-\tau)$ and $\mu=\tau$, so that

$$
\psi_{1}(t)=\frac{1}{\tau} \Theta(\tau-t)
$$

is the uniform distribution between 0 and $\tau$. Here $\Theta$ is the Heaviside function.

The Laplace transform of the correlation function can be calculated:

$$
\hat{C}(s)=a^{2}\left[\frac{1}{s}-\frac{(1-\Delta)\left(1-e^{-s \tau}\right)}{\tau s^{2}\left(1-\Delta e^{-s \tau}\right)}\right],
$$

where

$$
\Delta \equiv p-q
$$

This parameter measures the degree of correlation of the noise, as explained below. $\hat{C}(s)$ can be inverted to obtain 


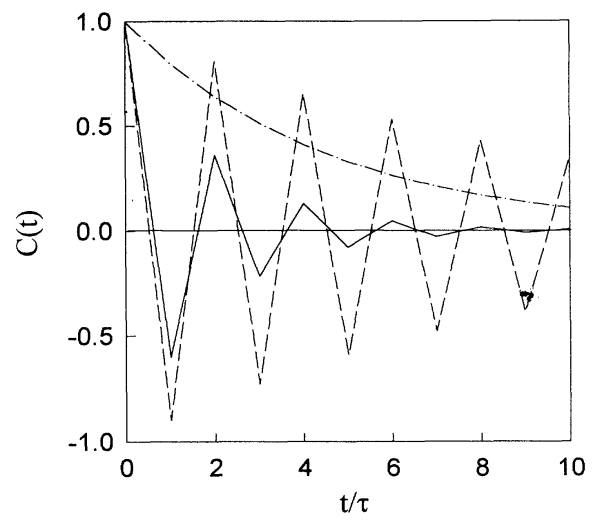

FIG. 5. Correlation function $C(t)$ for different values of $\Delta$ : $\Delta=-0.6$ (solid line), $\Delta=-0.8$ (dashed line), and $\Delta=0.8$ (dot-dashed line).

$$
C(t)=a^{2} \Delta^{N}[1-(1-\Delta)(t / \tau-N)]
$$

for $N \tau \leq t \leq(N+1) \tau$ with integer $N$. The correlation function oscillates between positive and negative values when $\Delta<0$ (that is, when the signal has a tendency to change its value after every time interval $\tau$ ) and remains positive when $\Delta>0$ (that is, when the signal tends to retain its value). The noise is not correlated for times longer than $\tau$ when $\Delta=0$. In Fig. $5, C(t)$ is shown for three different values of $\Delta$.

The correlation time $\tau_{c}$ gives an idea of the time interval over which the noisy signal is correlated. Among the various possible definitions of $\tau_{c}$ we choose [11]

$$
\tau_{c}=\frac{1}{C(0)} \int_{0}^{\infty} d t|C(t)| .
$$

Upon substitution of Eq. (A5) into this definition we obtain

$$
\tau_{c}=\left\{\begin{array}{lc}
\frac{\tau}{2}\left(\frac{1+\Delta}{1-\Delta}\right), & \Delta>0 \\
\frac{\tau}{2}\left(\frac{1+\Delta^{2}}{1-\Delta^{2}}\right), & \Delta<0
\end{array}\right.
$$

The correlation time increases as $|\Delta|$ increases (regardless of the sign of $\Delta$ ); this is reasonable since a greater

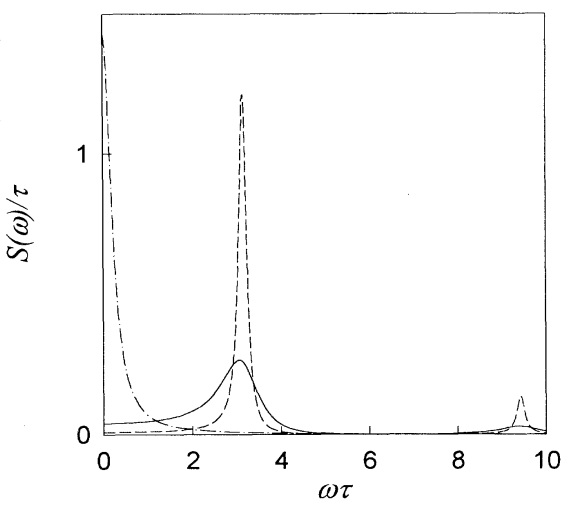

FIG. 6. Spectral density $S(\omega)$ for different values of $\Delta$ : $\Delta=-0.6$ (solid line), $\Delta=-0.8$ (dashed line), and $\Delta=0.8$ (dot-dashed line).

deviation of $\Delta$ from zero increases the predictability of the behavior of the signal [12].

A useful quantity is the spectral density $S(\omega)$, the Fourier transform of the autocorrelation function [13]. For the random signal considered here the spectral density reads

$$
S(\omega)=\frac{\left(1-\Delta^{2}\right)[1-\cos (\omega \tau)]}{\pi \tau \omega^{2}\left[1+\Delta^{2}-2 \Delta \cos (\omega \tau)\right]}
$$

It is worthwhile to mention that when $\Delta \rightarrow-1$, i.e., when $q \rightarrow 1$, the spectral density tends to concentrate around $\omega=\pi / \tau$ and its odd multiples. In this limit the noise becomes a nearly periodic signal of period $2 \tau$, giving rise to a periodic correlation function. In Fig. 6 the spectral density is shown for three values of $q$.

In addition to the limit described above, the coin-toss square wave has other interesting limits [7]. When $q \rightarrow 0$, it is a constant signal, retaining forever its initial value. If $\tau \rightarrow 0$ while $p=1-\lambda \tau$ and $q=\lambda \tau$, that is, $p$ goes to 1 and $q$ to 0 , the random signal converges in distribution to a Markovian dichotomous noise. $\lambda$ is the average time that the noise retains its value. Finally, if $a \rightarrow \infty$ and $\tau \rightarrow 0$ while $D=a^{2} \tau / 2$ remains fixed and finite, the noise converges to white noise of intensity $D_{r}=D p / q$ and $\left\langle\eta(t) \eta\left(t^{\prime}\right)\right\rangle=2 D_{r} \delta\left(t-t^{\prime}\right)$.
[1] D. R. Cox, Renewal Theory (Wiley, New York, 1962).

[2] A. J. Irwin, S. J. Fraser, and R. Kapral, Phys. Rev. Lett. 64, 2343 (1990); S. J. Fraser and R. Kapral, Phys. Rev. A 45, 3412 (1992).

[3] J. Stark, Phys. Rev. Lett. 65, 3357 (1990), and references therein.

[4] A. R. Cohen, IRE Trans. Circuit Theory CT-9, 371 (1962).

[5] J. M. Gutierrez, A. Iglesias, and M. A. Rodríguez, Phys. Rev. E 48, 2507 (1993).

[6] U. Behn, J. L. van Hemmen, R. Kühn, A. Lange, and V.
A. Zagrebnov, in On Three Levels, edited by M. Fannes, C. Maes, and A. Verbeure (Plenum, New York, 1994), p. 399.

[7] J. M. Porrà, J. Masoliver, and K. Lindenberg, Phys. Rev. E 48, 951 (1993).

[8] G. H. Weiss and R. J. Rubin, Adv. Chem. Phys. 52, 363 (1983).

[9] R. F. Pawula, J. M. Porrà, and J. Masoliver, Phys. Rev. E 47, 189 (1993).

[10] This result is deduced from Eq. (3.20) in [7].

[11] R. L. Stratonovich, Topics in the Theory of Random 
Noise (Gordon \& Breach, New York, 1963), Vol. 1.

[12] See [5], where the correlation time $\tau_{c}$ was defined as the average duration of the most probable sequence of the noise.
[13] C. W. Gardiner, Handbook of Stochastic Methods for Physics, Chemistry and the Natural Sciences (SpringerVerlag, Berlin, 1983). 